PROCEEDINGS OF THE

AMERICAN MATHEMATICAL SOCIETY

Volume 132, Number 4, Pages 1029-1035

S 0002-9939(03)07291-5

Article electronically published on December 1, 2003

\title{
SEBESTYÉN MOMENT PROBLEM: THE MULTI-DIMENSIONAL CASE
}

\author{
DAN POPOVICI AND ZOLTÁN SEBESTYÉN
}

(Communicated by Joseph A. Ball)

To the memory of Gyula Farkas

\begin{abstract}
Given a family $\left\{h_{\mathbf{n}}\right\}_{\mathbf{n} \in \mathbb{Z}_{+}^{\Omega}}$ of vectors in a Hilbert space $\mathcal{H}$ we characterize the existence of a family of commuting contractions $\mathbf{T}=\left\{T_{\omega}\right\}_{w \in \Omega}$ on $\mathcal{H}$ having regular dilation and such that

$$
h_{\mathbf{n}}=\mathbf{T}^{\mathbf{n}} h_{\mathbf{0}}, \quad \mathbf{n} \in \mathbb{Z}_{+}^{\Omega} .
$$

The theorem is a multi-dimensional analogue for some well-known operator moment problems due to Sebestyén in case $|\Omega|=1$ or, recently, to Găvruţă and Păunescu in case $|\Omega|=2$.
\end{abstract}

Let $\mathcal{H}$ be a complex Hilbert space. Denote by $\mathcal{L}(\mathcal{H})$ the $\mathbf{C}^{*}$-algebra of bounded linear operators on $\mathcal{H}$.

Z. Sebestyén proposed in $[5]$ the following operator moment problem:

Problem A. Given a sequence $\left\{h_{n}\right\}_{n \geq 0}$ of vectors in $\mathcal{H}$, under what condition does there exist a contraction $T$ on $\mathcal{H}$ such that

$$
h_{n}=T^{n} h_{0}, \quad n \geq 0 ?
$$

The two-dimensional analogue of this problem was proposed by Găvruţă and Păunescu [2]:

Problem B. Given a doubly indexed sequence $\left\{h_{m}^{n}\right\}_{m, n \geq 0}$ of vectors in $\mathcal{H}$, under what condition does there exist a commuting pair of contractions $(S, T)$ on $\mathcal{H}$ such that

$$
h_{m}^{n}=S^{m} T^{n} h_{0}^{0}, \quad m, n \geq 0 ?
$$

The solution given by Z. Sebestyén to the first problem was the following:

Theorem A ([5]). Let $\left\{h_{n}\right\}_{n \geq 0} \subset \mathcal{H}$. Problem $A$ has a solution if and only if

$$
\left\|\sum_{m, m^{\prime}} c_{m, m^{\prime}} h_{m+m^{\prime}}\right\|^{2} \leq \sum_{m, m^{\prime}, n, n^{\prime}} c_{m, m^{\prime}} \overline{c_{n, n^{\prime}}}\left\langle h_{(m-n)^{++}+m^{\prime}}, h_{(m-n)^{-}+n^{\prime}}\right\rangle,
$$

for every finite double sequence $\left\{c_{n, n^{\prime}}\right\}_{n, n^{\prime} \geq 0}$ of complex numbers (we used, for any integer $m$, the notation $m^{+}=\max \{m, 0\}$ and $\left.m^{-}=-\min \{m, 0\}\right)$.

Received by the editors October 22, 2002.

2000 Mathematics Subject Classification. Primary 47A57, 47A20.

Key words and phrases. Sebestyén operator moment problem, multi-contraction, regular dilation, positive definite function. 
The solution to the second problem uses the theory of regular dilations:

Theorem B ([2]). Let $\left\{h_{m}^{n}\right\}_{m, n \geq 0}$ be a sequence of vectors that spans $\mathcal{H}$. Problem $B$ has a solution $(S, T)$ having regular dilation if and only if

$$
\left\|\sum_{m, m^{\prime}, n, n^{\prime}} c_{m, m^{\prime}}^{n, n^{\prime}} h_{m+m^{\prime}}^{n+n^{\prime}}\right\|^{2} \leq \sum_{\substack{m, n, p, q \\ m^{\prime}, n^{\prime}, p^{\prime}, q^{\prime}}} c_{m, m^{\prime}}^{n, n^{\prime}} \overline{c_{p, p^{\prime}}^{q, q^{\prime}}}\left\langle h_{(m-p)^{+}+m^{\prime}}^{(n-q)^{+}+n^{\prime}}, h_{(m-p)^{-}+p^{\prime}}^{(n-q)^{-}+q^{\prime}}\right\rangle,
$$

for every finite double sequence $\left\{c_{m, m^{\prime}}^{n, n^{\prime}}\right\}_{m, m^{\prime}, n, n^{\prime} \geq 0}$ of complex numbers and the following regularity condition holds:

$$
\left\|h_{m+1}^{n}\right\|^{2}+\left\|h_{m}^{n+1}\right\|^{2} \leq\left\|h_{m}^{n}\right\|^{2}+\left\|h_{m+1}^{n+1}\right\|^{2}, \quad m, n \geq 0 .
$$

Before we formulate our multi-dimensional analogue of the previous moment problems we need to introduce some notions.

Let $\Omega$ be a nonempty set. Introduce $\mathbb{Z}^{\Omega}$ (respectively $\mathbb{Z}_{+}^{\Omega}$ ) as the set of $\mathbb{Z}$-valued (respectively $\mathbb{Z}_{+}$-valued) functions on $\Omega$ having finite support. With pointwise defined operation, $\mathbb{Z}^{\Omega}$ becomes an additive abelian group with unit $\mathbf{0}$ (the null function) having $\mathbb{Z}_{+}^{\Omega}$ as a subsemigroup. We identify some particular elements of $\mathbb{Z}_{+}^{\Omega}$ : for $\mathbf{m}=\left\{m_{\omega}\right\}_{\omega \in \Omega}$, let $\mathbf{m}^{+}=\left\{m_{\omega}^{+}\right\}_{\omega \in \Omega}$ and $\mathbf{m}^{-}=\left\{m_{\omega}^{-}\right\}_{\omega \in \Omega}$; for any finite subset $v \subset \Omega$, let $e(v)=\left\{\varphi_{v}(\omega)\right\}_{\omega \in \Omega}, \varphi_{v}$ being the characteristic function of $v$ on $\Omega$.

In what follows the term multi-contraction will denote a family $\mathbf{T}=\left\{T_{\omega}\right\}_{\omega \in \Omega}$ of commuting contractions on $\mathcal{H}$. If $\mathbf{T}=\left\{T_{\omega}\right\}_{\omega \in \Omega}$ is a multi-contraction and $\mathbf{m}=\left\{m_{\omega}\right\}_{\omega \in \Omega} \in \mathbb{Z}_{+}^{\Omega}$ we define, as usual, $\mathbf{T}^{\mathbf{m}}:=\prod_{\omega \in \Omega} T_{\omega}^{m_{\omega}}$. A multi-unitary operator $\mathbf{U}$ (multi-contraction consisting of unitary operators) acting on a Hilbert space $\mathcal{K} \supset \mathcal{H}$ is said to be a unitary dilation of a multi-contraction $\mathbf{T}$ if

$$
\mathbf{T}^{\mathbf{m}} h=P_{\mathcal{H}} \mathbf{U}^{\mathbf{m}} h, \quad \mathbf{m} \in \mathbb{Z}_{+}^{\Omega}, h \in \mathcal{H},
$$

$P_{\mathcal{H}}$ being the orthogonal projection of $\mathcal{K}$ onto $\mathcal{H}$. $\mathbf{U}$ is called regular if, moreover,

$$
\left(\mathbf{T}^{\mathbf{m}^{-}}\right)^{*} \mathbf{T}^{m^{+}} h=P_{\mathcal{H}} \mathbf{U}^{\mathbf{m}} h, \quad \mathbf{m} \in \mathbb{Z}^{\Omega}, h \in \mathcal{H}
$$

holds. Here $\mathbf{U}$ is multi-unitary; so the definition of $\mathbf{U}^{\mathbf{m}}$ can be obviously extended to all of $\mathbf{m} \in \mathbb{Z}^{\Omega}$.

Remark. Regular dilations have been introduced and studied by S. Brehmer [1]. We should also mention here the paper of B. Sz.-Nagy [ $\underline{6}$, which brought more light on the subject.

By the classical theorem of M. A. Neumark [4] on positive definite functions on a group (see also [7]) we can state that a multi-contraction $\mathbf{T}$ has regular dilation if and only if the operator-valued function

$$
\mathbb{Z}^{\Omega} \ni \mathbf{m} \mapsto T(\mathbf{m}):=\left(\mathbf{T}^{\mathbf{m}^{-}}\right)^{*} \mathbf{T}^{\mathbf{m}^{+}} \in \mathcal{L}(\mathcal{H})
$$

is positive definite on $\mathbb{Z}^{\Omega}$, i.e.,

$$
\sum_{\mathbf{m}, \mathbf{n} \in \mathbb{Z}_{+}^{\Omega}}\left\langle T(\mathbf{n}-\mathbf{m}) h_{\mathbf{n}}, h_{\mathbf{m}}\right\rangle \geq 0,
$$

for any finite family $\left\{h_{\mathbf{n}}\right\}_{\mathbf{n} \in \mathbb{Z}_{+}^{\Omega}}$ of vectors in $\mathcal{H}$.

Equivalently ([1]; cf. also 3], [7]), T has regular dilation if and only if

$$
\sum_{v \subset u}(-1)^{|v|}\left(T^{e(v)}\right)^{*} T^{e(v)} \geq 0,
$$


for any finite subset $u$ of $\Omega$.

Our terminology and approach in the following will be close to the one used in the monograph [7].

We are now ready to formulate the multi-dimensional analogue of the Sebestyén operator moment problem (Problem A):

Problem C. Given a family $\left\{h_{\mathbf{n}}\right\}_{\mathbf{n} \in \mathbb{Z}_{+}^{\Omega}}$ of vectors in $\mathcal{H}$, under what condition does there exist a multi-contraction $\mathbf{T}$ on $\mathcal{H}$ such that

$$
h_{\mathbf{n}}=\mathbf{T}^{\mathbf{n}} h_{\mathbf{0}}, \quad \mathbf{n} \in \mathbb{Z}_{+}^{\Omega} ?
$$

Our solution applies to the theory of regular dilations (more precisely the results stated in the Remark) and also naturally extends the methods in [5].

Lemma. Let $\left\{c_{\mathbf{n}, \mathbf{n}^{\prime}}\right\}_{\mathbf{n}, \mathbf{n}^{\prime} \in \mathbb{Z}_{+}^{\Omega}}$ and $\left\{d_{\mathbf{n}, \mathbf{n}^{\prime}}\right\}_{\mathbf{n}, \mathbf{n}^{\prime} \in \mathbb{Z}_{+}^{\Omega}}$ be given families of complex numbers. The following conditions are equivalent:

(i)

$$
\sum_{\mathbf{n}^{\prime}} c_{\mathbf{n}, \mathbf{n}^{\prime}} h_{\mathbf{n}^{\prime}+\mathbf{p}}=\sum_{\substack{\mathbf{n}^{\prime} \in \mathbb{Z}_{+}^{\Omega} \\ v \subset \Omega \text { finite }}}(-1)^{|v|} d_{\mathbf{n}+e(v), \mathbf{n}^{\prime}} h_{\mathbf{n}^{\prime}+e(v)+\mathbf{p}}, \quad \mathbf{n}, \mathbf{p} \in \mathbb{Z}_{+}^{\Omega},
$$

for any family $\left\{h_{\mathbf{n}}\right\}_{\mathbf{n} \in \mathbb{Z}_{+}^{\Omega}} \subset \mathcal{H}$ with finite support;

(ii)

$$
\sum_{\mathbf{m} \geq \mathbf{n} ; \mathbf{m}^{\prime}} c_{\mathbf{m}, \mathbf{m}^{\prime}} h_{\mathbf{m}+\mathbf{m}^{\prime}-\mathbf{n}+\mathbf{p}}=\sum_{\mathbf{n}^{\prime}} d_{\mathbf{n}, \mathbf{n}^{\prime}} h_{\mathbf{n}^{\prime}+\mathbf{p}}, \quad \mathbf{n}, \mathbf{p} \in \mathbb{Z}_{+}^{\Omega},
$$

for any family $\left\{h_{\mathbf{n}}\right\}_{\mathbf{n} \in \mathbb{Z}_{+}^{\Omega}} \subset \mathcal{H}$ with finite support;

(iii)

$$
c_{\mathbf{n}, \mathbf{n}^{\prime}}=\sum_{v: e(v) \leq \mathbf{n}^{\prime}}(-1)^{|v|} d_{\mathbf{n}+e(v), \mathbf{n}^{\prime}-e(v)}, \quad \mathbf{n}, \mathbf{n}^{\prime} \in \mathbb{Z}_{+}^{\Omega}
$$

$(i v)$

$$
d_{\mathbf{n}, \mathbf{n}^{\prime}}=\sum_{\mathbf{q} \leq \mathbf{n}^{\prime}} c_{\mathbf{n}+\mathbf{q}, \mathbf{n}^{\prime}-\mathbf{q}}, \quad \mathbf{n}, \mathbf{n}^{\prime} \in \mathbb{Z}_{+}^{\Omega} .
$$

Moreover, $\left\{c_{\mathbf{n}, \mathbf{n}^{\prime}}\right\}$ has finite support if and only if $\left\{d_{\mathbf{n}, \mathbf{n}^{\prime}}\right\}$ has finite support.

Proof. $($ i $) \Leftrightarrow($ iii $)$ and $(i i) \Leftrightarrow($ iv $)$ are easy observations (just compute the coefficient of each $\left.h_{\mathbf{n}}, \mathbf{n} \in \mathbb{Z}_{+}^{\Omega}\right)$.

For $(i i i) \Leftrightarrow(i v)$ observe firstly that

$$
\begin{aligned}
& \sum_{\mathbf{q} \leq \mathbf{n}^{\prime}} \sum_{v: e(v) \leq \mathbf{n}^{\prime}-\mathbf{q}}(-1)^{|v|} d_{\mathbf{n}+\mathbf{q}+e(v), \mathbf{n}^{\prime}-\mathbf{q}-e(v)} \\
& \quad \stackrel{\mathbf{q}+e(v)=\mathbf{p}}{=} \sum_{\mathbf{p}: \mathbf{p} \leq \mathbf{n}^{\prime}} \sum_{v: e(v) \leq \mathbf{p}}(-1)^{|v|} d_{\mathbf{n}+\mathbf{p}, \mathbf{n}^{\prime}-\mathbf{p}}=d_{\mathbf{n}, \mathbf{n}^{\prime}}, \quad \mathbf{n}, \mathbf{n}^{\prime} \in \mathbb{Z}_{+}^{\Omega},
\end{aligned}
$$


since $\sum_{v: e(v) \leq \mathbf{p}}(-1)^{|v|}=0$ for $\mathbf{p}=\mathbf{0}$ or 1 for $\mathbf{p} \neq \mathbf{0}$. Conversely,

$$
\begin{aligned}
\sum_{v: e(v) \leq \mathbf{n}^{\prime}}(-1)^{|v|} & \sum_{\substack{\mathbf{q} \leq \mathbf{n}^{\prime}-e(v) \\
\mathbf{q}+e(v)=\mathbf{p}}} c_{\mathbf{n}+e(v)+\mathbf{q}, \mathbf{n}^{\prime}-e(v)-\mathbf{q}} \sum_{v: e(v) \leq \mathbf{n}^{\prime}}(-1)^{|v|} \sum_{\mathbf{p}: e(v) \leq \mathbf{p} \leq \mathbf{n}^{\prime}} c_{\mathbf{n}+\mathbf{p}, \mathbf{n}^{\prime}-\mathbf{p}} \\
& =\sum_{\mathbf{p} \leq \mathbf{n}^{\prime}} c_{\mathbf{n}+\mathbf{p}, \mathbf{n}^{\prime}-\mathbf{p}} \sum_{v: e(v) \leq \mathbf{p}}(-1)^{|v|}=c_{\mathbf{n}, \mathbf{n}^{\prime}}, \quad \mathbf{n}, \mathbf{n}^{\prime} \in \mathbb{Z}_{+}^{\Omega},
\end{aligned}
$$

by the same argument as above.

The solution for Problem C now follows:

Theorem C. Let $\left\{h_{\mathbf{n}}\right\}_{\mathbf{n} \in \mathbb{Z}_{+}^{\Omega}}$ be a given multi-indexed family of vectors in $\mathcal{H}$. The following conditions are equivalent:

(a) Problem $C$ has a solution $\mathbf{T}=\left\{T_{\omega}\right\}_{\omega \in \Omega}$ having regular dilation;

(b)

$$
\left\|\sum_{\mathbf{m}, \mathbf{m}^{\prime}} c_{\mathbf{m}, \mathbf{m}^{\prime}} h_{\mathbf{m}+\mathbf{m}^{\prime}}\right\|^{2} \leq \sum_{\mathbf{m}, \mathbf{m}^{\prime}, \mathbf{n}, \mathbf{n}^{\prime}} c_{\mathbf{m}, \mathbf{m}^{\prime}} \overline{c_{\mathbf{n}, \mathbf{n}^{\prime}}}\left\langle h_{(\mathbf{m}-\mathbf{n})^{+}+\mathbf{m}^{\prime}}, h_{(\mathbf{m}-\mathbf{n})^{-}+\mathbf{n}^{\prime}}\right\rangle,
$$

for every finite family $\left\{c_{\mathbf{n}, \mathbf{n}^{\prime}}\right\}_{\mathbf{n}, \mathbf{n}^{\prime} \in \mathbb{Z}_{+}^{\Omega}}$ of complex numbers;

(c)

$$
\sum_{\mathbf{m}, \mathbf{m}^{\prime}, \mathbf{n}, \mathbf{n}^{\prime}} c_{\mathbf{m}, \mathbf{m}^{\prime}} \overline{C_{\mathbf{n}, \mathbf{n}^{\prime}}}\left\langle h_{(\mathbf{m}-\mathbf{n})^{+}+\mathbf{m}^{\prime}}, h_{(\mathbf{m}-\mathbf{n})^{-}+\mathbf{n}^{\prime}}\right\rangle \geq 0,
$$

for every finite family $\left\{c_{\mathbf{n}, \mathbf{n}^{\prime}}\right\}_{\mathbf{n}, \mathbf{n}^{\prime} \in \mathbb{Z}_{+}^{\Omega}}$ of complex numbers;

(d)

$$
\sum_{v \subset u}(-1)^{|v|}\left\|\sum_{\mathbf{n}} c_{\mathbf{n}} h_{\mathbf{n}+e(v)}\right\|^{2} \geq 0
$$

for every finite subset $u \subset \Omega$ and finite family $\left\{c_{\mathbf{n}}\right\}_{\mathbf{n} \in \mathbb{Z}_{+}^{\Omega}}$ of complex numbers.

Proof. $(b) \Rightarrow(c)$ is obvious. Replace $\left\{c_{\mathbf{n}, \mathbf{n}^{\prime}}\right\}$ in $(c)$ by the finite family $\left\{d_{\mathbf{n}, \mathbf{n}^{\prime}}\right\}$ given by the previous Lemma (iii). The inequality in $(c)$ becomes

$$
\begin{aligned}
\sum_{\mathbf{m}, \mathbf{n}}\left\langle\sum_{v, \mathbf{m}^{\prime}}(-1)^{|v|} d_{\mathbf{m}+e(v), \mathbf{m}^{\prime}} h_{\mathbf{m}^{\prime}+e(v)+(\mathbf{m}-\mathbf{n})^{+},}\right. & \\
& \left.\sum_{w, \mathbf{n}^{\prime}}(-1)^{|w|} d_{\mathbf{n}+e(w), \mathbf{n}^{\prime}} h_{\mathbf{n}^{\prime}+e(w)+(\mathbf{m}-\mathbf{n})^{-}}\right\rangle \geq 0,
\end{aligned}
$$

by the same Lemma $(i)$. Change variables $\mathbf{m} \leftrightarrow \mathbf{m}+e(v)$ and $\mathbf{n} \leftrightarrow \mathbf{n}+e(w)$ to obtain

$$
\begin{aligned}
\sum_{\mathbf{m}, \mathbf{m}^{\prime}, \mathbf{n}, \mathbf{n}^{\prime}} \sum_{\begin{array}{r}
v: e(v) \leq \mathbf{m} \\
w: e(w) \leq \mathbf{n}
\end{array}}(-1)^{|v|+|w|} d_{\mathbf{m}, \mathbf{m}^{\prime}} \overline{d_{\mathbf{n}, \mathbf{n}^{\prime}}}\left\langle h_{\mathbf{m}^{\prime}+e(v)+(\mathbf{m}-e(v)-\mathbf{n}+e(w))^{+},}\right. \\
\left.h_{\mathbf{n}^{\prime}+e(w)+(\mathbf{m}-e(v)-\mathbf{n}+e(w))^{-}}\right\rangle \geq 0 .
\end{aligned}
$$

For $\mathbf{p}=\left\{p_{\omega}\right\}_{\omega \in \Omega} \in \mathbb{Z}^{\Omega}$, define $\pi(\mathbf{p})=\left\{\omega \in \Omega \mid p_{\omega}>0\right\}$. Any finite set $v \subset \Omega$ with $e(v) \leq \mathbf{m}$ (or equivalently $v \subset \pi(\mathbf{m})$ ) can be partitioned by $v=v^{\prime} \cup v^{\prime \prime}$ with $v^{\prime} \subset \pi(\mathbf{m}) \backslash \pi(\mathbf{m}-\mathbf{n}+e(w))$ and $v^{\prime \prime} \subset \pi(\mathbf{m}) \cap \pi(\mathbf{m}-\mathbf{n}+e(w))$. 
Observe that, for $\mathbf{m} \neq \mathbf{n}$ ( suppose $(\mathbf{m}-\mathbf{n})^{+} \neq \mathbf{0}$, i.e., $\left.\pi(\mathbf{m}-\mathbf{n}) \neq \emptyset\right)$ and fixed $\mathbf{m}^{\prime}, \mathbf{n}^{\prime}, w$ :

$$
\begin{aligned}
& \sum_{v \subset \pi(\mathbf{m})}(-1)^{|v|}\left\langle h_{\mathbf{m}^{\prime}+e(v)+(\mathbf{m}-e(v)-\mathbf{n}+e(w))^{+}}, h_{\mathbf{n}^{\prime}+e(w)+(\mathbf{m}-e(v)-\mathbf{n}+e(w))^{-}}\right\rangle \\
& =\sum_{v^{\prime} \subset \pi(\mathbf{m}) \backslash \pi(\mathbf{m}-\mathbf{n}+e(w))}(-1)^{\left|v^{\prime}\right|} \sum_{v^{\prime \prime} \subset \pi(\mathbf{m}) \cap \pi(\mathbf{m}-\mathbf{n}+e(w))}(-1)^{\left|v^{\prime \prime}\right|}
\end{aligned}
$$

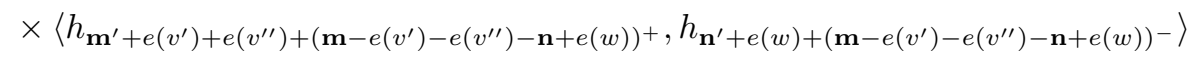

$$
\begin{aligned}
& =\sum_{v^{\prime} \subset \pi(\mathbf{m}) \backslash \pi(\mathbf{m}-\mathbf{n}+e(w))}(-1)^{\left|v^{\prime}\right|}\left[\sum_{v^{\prime \prime} \subset \pi(\mathbf{m}) \cap \pi(\mathbf{m}-\mathbf{n}+e(w))}(-1)^{\left|v^{\prime \prime}\right|}\right] \\
& \times\left\langle h_{\mathbf{m}^{\prime}+e\left(v^{\prime}\right)+\left(m-e\left(v^{\prime}\right)-\mathbf{n}+e(w)\right)^{+}}, h_{\mathbf{n}^{\prime}+e(w)+\left(\mathbf{m}-e\left(v^{\prime}\right)-\mathbf{n}+e(w)\right)^{-}}\right\rangle=0,
\end{aligned}
$$

the term in brackets being null $(\pi(\mathbf{m}) \cap \pi(\mathbf{m}-\mathbf{n}+e(w))$ contains $\pi(\mathbf{m}-\mathbf{n})$, which is non-void).

By this observation the positivity condition (3) becomes

$$
\sum_{\mathbf{m}, \mathbf{m}^{\prime}, \mathbf{n}^{\prime}} \sum_{v, w \subset \pi(\mathbf{m})}(-1)^{|v|+|w|} d_{\mathbf{m}, \mathbf{m}^{\prime}} \overline{d_{\mathbf{m}, \mathbf{n}^{\prime}}}\left\langle h_{\mathbf{m}^{\prime}+e(v \cup w)}, h_{\mathbf{n}^{\prime}+e(v \cup w)}\right\rangle \geq 0
$$

or, equivalently,

$$
\sum_{\mathbf{m}}\left[\sum_{u \subset \pi(\mathbf{m})}(-1)^{|u|}\left\|\sum_{\mathbf{m}^{\prime}} d_{\mathbf{m}, \mathbf{m}^{\prime}} h_{\mathbf{m}^{\prime}+e(u)}\right\|^{2}\right] \geq 0
$$

since $\sum_{v, w: v \cup w=u}(-1)^{|v|+|w|}=(-1)^{|u|}$.

The family $\left\{d_{\mathbf{m}, \mathbf{m}^{\prime}}\right\}$ with finite support being arbitrary, every sum in the brackets above must be positive or zero. The implication $(c) \Rightarrow(d)$ is proved.

$(d) \Rightarrow(b)$ Rewrite $(d)$ in the form:

$$
\sum_{\mathbf{m} \geq 0}\left[\sum_{u \subset \pi(\mathbf{m})}(-1)^{|u|}\left\|\sum_{\mathbf{m}^{\prime}} d_{\mathbf{m}, \mathbf{m}^{\prime}} h_{\mathbf{m}^{\prime}+e(u)}\right\|^{2}\right] \geq\left\|\sum_{\mathbf{m}^{\prime}} d_{\mathbf{0}, \mathbf{m}^{\prime}} h_{\mathbf{m}^{\prime}}\right\|^{2},
$$

for any finite subset $u \subset \Omega$ and finite family $\left\{d_{\mathbf{m}, \mathbf{m}^{\prime}}\right\}$ of complex numbers. Replace $\left\{d_{\mathbf{m}, \mathbf{m}^{\prime}}\right\}$ by the finite family $\left\{c_{\mathbf{m}, \mathbf{m}^{\prime}}\right\}$ given by Lemma $(i v)$. Similar calculations as in the previous implication and further use of the Lemma lead us to condition $(b)$.

$(a) \Rightarrow(d)$ By $(a)$ the solution to Problem $\mathrm{C}$ is a multi-contraction $\mathbf{T}$ having regular dilation. Use the equalities $h_{\mathbf{n}}=\mathbf{T}^{\mathbf{n}} h_{\mathbf{0}}, \mathbf{n} \in \mathbb{Z}_{+}^{\Omega}$ in (2) to finally obtain $(d)$.

$(b) \Rightarrow(a)$ Let $\mathcal{F}_{0}$ be the linear space of all finite families $\left\{c_{\mathbf{n}, \mathbf{n}^{\prime}}\right\}_{\mathbf{n}, \mathbf{n}^{\prime} \in \mathbb{Z}_{+}^{\Omega}}$ of complex numbers. Endow $\mathcal{F}_{0}$ with the semi-inner product (in view of $(b)$ ):

$$
\left\langle\left\{c_{\mathbf{n}, \mathbf{n}^{\prime}}\right\},\left\{d_{\mathbf{n}, \mathbf{n}^{\prime}}\right\}\right\rangle_{\mathcal{F}_{0}}:=\sum_{\mathbf{m}, \mathbf{m}^{\prime}, \mathbf{n}, \mathbf{n}^{\prime}} c_{\mathbf{m}, \mathbf{m}^{\prime}} \overline{d_{\mathbf{n}, \mathbf{n}^{\prime}}}\left\langle h_{(\mathbf{m}-\mathbf{n})^{+}+\mathbf{m}^{\prime}}, h_{(\mathbf{m}-\mathbf{n})^{-}+\mathbf{n}^{\prime}}\right\rangle .
$$

Factorize $\mathcal{F}_{0}$ with respect to the null space of $\langle\cdot, \cdot\rangle_{\mathcal{F}_{0}}$. Denote by $\mathcal{F}$ its Hilbert space completion and by $\mathcal{H}^{\prime}$ the span of $\left\{h_{\mathbf{n}}\right\}_{\mathbf{n} \in \mathbb{Z}_{+}^{\Omega}}$.

Define $V: \mathcal{F} \rightarrow \mathcal{H}^{\prime}$,

$$
V\left\{c_{\mathbf{n}, \mathbf{n}^{\prime}}\right\}:=\sum_{\mathbf{n}, \mathbf{n}^{\prime}} c_{\mathbf{n}, \mathbf{n}^{\prime}} h_{\mathbf{n}+\mathbf{n}^{\prime}}, \quad\left\{c_{\mathbf{n}, \mathbf{n}^{\prime}}\right\} \in \mathcal{F}_{0} .
$$


By $(a), V$ can be extended to a contraction on $\mathcal{F}$ into $\mathcal{H}^{\prime}$. Moreover, by easy computations,

$$
V^{*} h_{\mathbf{k}}=\left\{\delta_{\left(\mathbf{n}, \mathbf{n}^{\prime}\right)}^{(\mathbf{0}, \mathbf{k})}\right\}_{\mathbf{n}, \mathbf{n}^{\prime}} \in \mathbb{Z}_{+}^{\Omega}, \quad \mathbf{k} \in \mathbb{Z}_{+}^{\Omega}
$$

( $\delta$ denotes here the Kronecker symbol).

For fixed $\omega \in \Omega$, define $U_{\omega}$ on $\mathcal{F}_{0}$ by

$$
U_{\omega}\left\{c_{\mathbf{n}, \mathbf{n}^{\prime}}\right\}_{\mathbf{n}, \mathbf{n}^{\prime}}:=\left\{c_{\mathbf{n}+e(\{w\}), \mathbf{n}^{\prime}}\right\}_{\mathbf{n}, \mathbf{n}^{\prime}} .
$$

$U_{\omega}$ can be extended on $\mathcal{F}$, and it is not hard to see that its adjoint $U_{\omega}^{*}$ is isometric. Let

$$
T_{\omega}^{\prime}=V U_{\omega}^{*} V^{*} \in \mathcal{L}\left(\mathcal{H}^{\prime}\right) .
$$

Each $T_{\omega}$ is a contraction on $\mathcal{H}^{\prime}$. Moreover,

$$
T_{\omega}^{\prime} h_{\mathbf{k}}=V U_{\omega}^{*} V^{*} h_{\mathbf{k}}=V U_{\omega}^{*}\left\{\delta_{\left(\mathbf{n}, \mathbf{n}^{\prime}\right)}^{(\mathbf{0}, \mathbf{k})}\right\}_{\mathbf{n}, \mathbf{n}^{\prime}}=V\left\{\delta_{\left(\mathbf{n}, \mathbf{n}^{\prime}\right)}^{(e(\{w\}), \mathbf{k})}\right\}_{\mathbf{n}, \mathbf{n}^{\prime}}=h_{\mathbf{k}+e(\{w\})}, k \in \mathbb{Z}_{+}^{\Omega}
$$

and, consequently,

$$
T_{\omega}^{\prime} T_{\omega^{\prime}}^{\prime} h_{\mathbf{n}}=h_{\mathbf{n}+e\left(\left\{\omega, \omega^{\prime}\right\}\right)}=T_{\omega^{\prime}}^{\prime} T_{\omega}^{\prime} h_{\mathbf{n}}, \quad\left\{\omega, \omega^{\prime}\right\} \subset \Omega, \mathbf{n} \in \mathbb{Z}_{+}^{\Omega} .
$$

$\mathbf{T}^{\prime}=\left\{T_{\omega}^{\prime}\right\}_{\omega \in \Omega}$ is a multi-contraction on $\mathcal{H}^{\prime}$ and, since

$$
\mathbf{T}^{\prime} h_{\mathbf{0}}=h_{\sum_{\omega \in \Omega} n_{\omega} e(\{\omega\})}=h_{\mathbf{n}}, \quad \mathbf{n} \in \mathbb{Z}_{+}^{\Omega},
$$

$\mathbf{T}^{\prime}$ is a solution for Problem C. In addition, by $(c)$, for any $x_{\mathbf{m}}=\sum_{\mathbf{m}^{\prime}} c_{\mathbf{m}, \mathbf{m}^{\prime}} h_{\mathbf{m}^{\prime}} \in$ $\mathcal{H}^{\prime}\left(\mathbf{m} \in \mathbb{Z}_{+}^{\Omega}\right)$,

$$
\begin{aligned}
& \sum_{\mathbf{m}, \mathbf{n} \in \mathbb{Z}_{+}^{\Omega}}\left\langle\left[\mathbf{T}^{(\mathbf{m}-\mathbf{n})^{-}}\right]^{*} \mathbf{T}^{(\mathbf{m}-\mathbf{n})^{+}} x_{\mathbf{m}}, x_{\mathbf{n}}\right\rangle \\
& =\sum_{\mathbf{m}, \mathbf{n}}\left\langle\mathbf{T}^{\prime(\mathbf{m}-\mathbf{n})^{+}} x_{\mathbf{m}}, \mathbf{T}^{\prime(\mathbf{m}-\mathbf{n})^{-}} x_{\mathbf{n}}\right\rangle \\
& =\sum_{\mathbf{m}, \mathbf{m}^{\prime}, \mathbf{n}, \mathbf{n}^{\prime}} c_{\mathbf{m}, \mathbf{m}^{\prime}} \overline{\bar{n}_{\mathbf{n}, \mathbf{n}^{\prime}}}\left\langle\mathbf{T}^{\prime(\mathbf{m}-\mathbf{n})^{+}} h_{\mathbf{m}^{\prime}}, \mathbf{T}^{\prime(\mathbf{m}-\mathbf{n})^{-}} h_{\mathbf{n}^{\prime}}\right\rangle \\
& =\sum_{\mathbf{m}, \mathbf{m}^{\prime}, \mathbf{n}, \mathbf{n}^{\prime}} c_{\mathbf{m}, \mathbf{m}^{\prime}} \overline{\mathbf{c}_{\mathbf{n}, \mathbf{n}^{\prime}}}\left\langle h_{(\mathbf{m}-\mathbf{n})^{+}+\mathbf{m}^{\prime}}, h_{(\mathbf{m}-\mathbf{n})^{-}+\mathbf{n}^{\prime}}\right\rangle \geq 0 .
\end{aligned}
$$

The positivity condition (1) is then verified and $\mathbf{T}^{\prime}$ has a regular dilation. Extend $\mathbf{T}^{\prime}$ on $\mathcal{H}$ by $T_{\omega}=T_{\omega}^{\prime} \oplus 0_{\mathcal{H}^{\prime \prime}}(\omega \in \Omega)$ corresponding to the orthogonal decomposition $\mathcal{H}=\mathcal{H}^{\prime} \oplus \mathcal{H}^{\prime \prime} . \mathbf{T}=\left\{T_{\omega}\right\}_{\omega \in \Omega}$ is a multi-contraction on $\mathcal{H}$ that extends $\mathbf{T}^{\prime} . \mathbf{T}$ is still a solution of Problem $\mathrm{C}$ and, since

$$
\begin{aligned}
& \left\langle\sum_{v \subset u}(-1)^{|v|}\left(\mathbf{T}^{e(v)}\right)^{*} \mathbf{T}^{e(v)}\left(h^{\prime}+h^{\prime \prime}\right), h^{\prime}+h^{\prime \prime}\right\rangle \\
& =\sum_{v \subset u}(-1)^{|v|}\left\|\mathbf{T}^{\prime} e(v) h^{\prime}\right\|^{2}+\left\|h^{\prime}\right\|^{2}+\left\|h^{\prime \prime}\right\|^{2} \geq 0, \quad h^{\prime} \in \mathcal{H}^{\prime}, h^{\prime \prime} \in \mathcal{H}^{\prime \prime}, u \subset \Omega \text { finite, }
\end{aligned}
$$

$\mathbf{T}$ has regular dilation (following condition (2) for regularity).

In particular, for $\Omega$ with $|\Omega|=2$, we can improve the solution to Problem B. The condition that $\left\{h_{\mathbf{n}}\right\}_{\mathbf{n} \in \mathbb{Z}_{+}^{\Omega}}$ spans $\mathcal{H}$ is no longer necessary, and the regularity condition is actually a consequence of the positivity condition. More precisely, we have the following theorem. 
Theorem $\mathbf{B}+$. Let $\left\{h_{\mathbf{n}}\right\}_{\mathbf{n} \in \mathbb{Z}_{+}^{\Omega}}$ be a given sequence of vectors in $\mathcal{H}$. The following conditions are equivalent:

(a) Problem $B$ has a solution $\mathbf{T}=\left(T_{1}, T_{2}\right)$ having regular dilation;

(b)

$$
\left\|\sum_{\mathbf{m}, \mathbf{m}^{\prime}} c_{\mathbf{m}, \mathbf{m}^{\prime}} h_{\mathbf{m}+\mathbf{m}^{\prime}}\right\|^{2} \leq \sum_{\mathbf{m}, \mathbf{m}^{\prime}, \mathbf{n}, \mathbf{n}^{\prime}} c_{\mathbf{m}, \mathbf{m}^{\prime}} \overline{c_{\mathbf{n}, \mathbf{n}^{\prime}}}\left\langle h_{(\mathbf{m}-\mathbf{n})^{+}+\mathbf{m}^{\prime}}, h_{(\mathbf{m}-\mathbf{n})^{-}+\mathbf{n}^{\prime}}\right\rangle
$$

for every finite family $\left\{c_{\mathbf{n}, \mathbf{n}^{\prime}}\right\}_{\mathbf{n}, \mathbf{n}^{\prime} \in \mathbb{Z}_{+}^{2}}$ of complex numbers;

(c)

$$
\sum_{\mathbf{m}, \mathbf{m}^{\prime}, \mathbf{n}, \mathbf{n}^{\prime}} c_{\mathbf{m}, \mathbf{m}^{\prime}} \overline{C_{\mathbf{n}, \mathbf{n}^{\prime}}}\left\langle h_{(\mathbf{m}-\mathbf{n})^{+}+\mathbf{m}^{\prime}}, h_{(\mathbf{m}-\mathbf{n})^{-}+\mathbf{n}^{\prime}}\right\rangle \geq 0,
$$

for every finite family $\left\{c_{\mathbf{n}, \mathbf{n}^{\prime}}\right\}_{\mathbf{n}, \mathbf{n}^{\prime} \in \mathbb{Z}_{+}^{2}}$ of complex numbers;

(d)

$$
\begin{gathered}
\left\|\sum_{\mathbf{n}} c_{\mathbf{n}} h_{\mathbf{n}+e_{1}}\right\|^{2}+\left\|\sum_{\mathbf{n}} c_{\mathbf{n}} h_{\mathbf{n}+e_{2}}\right\|^{2} \leq\left\|\sum_{\mathbf{n}} c_{\mathbf{n}} h_{\mathbf{n}}\right\|^{2}+\left\|\sum_{\mathbf{n}} c_{\mathbf{n}} h_{\mathbf{n}+e_{1}+e_{2}}\right\|^{2}, \\
\left\|\sum_{\mathbf{n}} c_{\mathbf{n}} h_{\mathbf{n}+e_{1}}\right\| \leq\left\|\sum_{\mathbf{n}} c_{\mathbf{n}} h_{\mathbf{n}}\right\| \quad \text { and }\left\|\sum_{\mathbf{n}} c_{\mathbf{n}} h_{\mathbf{n}+e_{2}}\right\| \leq\left\|\sum_{\mathbf{n}} c_{\mathbf{n}} h_{\mathbf{n}}\right\|,
\end{gathered}
$$

for every finite family $\left\{c_{\mathbf{n}, \mathbf{n}^{\prime}}\right\}_{\mathbf{n}, \mathbf{n}^{\prime} \in \mathbb{Z}_{+}^{2}}$ of complex numbers (we use here $e_{1}=(1,0)$ and $\left.e_{2}=(0,1)\right)$.

\section{REFERENCES}

[1] S. Brehmer, Über vertauschbare Kontraktionen des Hilbertschen Raumes, Acta Sci. Math. Szeged 22 (1961), 106-111. MR 24:A1023a

[2] P. Găvruţă and D. Păunescu, Sebestyén's moment problem and regular dilations, Acta Math. Hungar. 94 (2002), 223-232. MR 2003g:47014

[3] I. Halperin, Sz.-Nagy - Brehmer dilations, Acta Sci. Math. Szeged 23 (1962), 279-289. MR 27:6127a

[4] M. A. Neumark, Positive definite operator functions on a commutative group, Bulletin Acad. Sci. URSS Sér. Math. 7 (1943), 237-244. MR 5:272C

[5] Z. Sebestyén, Moment theorems for operators on Hilbert space, Acta Sci. Math. Szeged 44 (1982), 165-171. MR 84f:47004

[6] B. Sz.-Nagy, Bemerkungen zur vorstehenden Arbeit des Herrn S. Brehmer, Acta Sci. Math. Szeged 22 (1961), 112-114. MR 24:A1023b

[7] B. Sz.-Nagy and C. Foiaş, Harmonic analysis of operators on Hilbert space, Akadémiai Kiadó, Budapest, North-Holland, Amsterdam, London, 1970. MR 43:947

Department of Mathematics, University of the West, Ro-1900 Timişoara, Bd. V. PÂRVAN 4, Romania

E-mail address: popovici@math.uvt.ro

Department of Applied Analysis, Loránd Eötvös University, H-1117 Budapest, PÁzmány PÉTer sétány 1/C, Hungary

E-mail address: sebesty@cs.elte.hu 